\title{
Immunolocalization of PPP1C Isoforms in SH-SY5Y Cells during the Cell Cycle
}

\author{
M. Santos*, S. Rebelo*, O. A. B. da Cruz e Silva* and E. F. da Cruz e Silva**† \\ * Neurosciences Laboratory, Centre for Cell Biology, University of Aveiro, Campus de Santiago, \\ 3810-193 Aveiro, Portugal \\ ** Signal Transduction Laboratory, Center for Cell Biology, Health Sciences and Biology \\ Department, University of Aveiro, Campus de Santiago, 3810-193 Aveiro \\ † Deceased
}

Phosphoprotein phosphatase 1 (PPP1) is the most widely expressed and abundant serine/threonine protein phosphatase. PPP1 regulates a variety of cellular functions such as glycogen metabolism, mitosis and meiosis, cell-cycle arrest, apoptosis, dynamics of actin cytoskeleton, protein synthesis among others [reviewed in 1]. The versatility of the PPP1 catalytic subunit (PPP1C) is achieved by associating with different regulatory subunits that target PPP1C to a particular subcellular compartment determining its substrate specificity and activity. PPP1C is expressed in mammals in three isoforms: PPP1CA, PPP1CB and PPP1CC, each encoded by distinct genes. The PPPIC gene undergoes alternative splicing to originate PPP1CC1 and PPP1CC2 variants. PPP1A and PPP1C1 are expressed virtually in all tissues but are particularly enriched in the brain. The subcellular localization of the endogenous PPP1C isoforms is not fully elucidated, but all isoforms are found in the nucleus and cytoplasm, despite some isoform-specific differences in intranuclear distribution. Furthermore, it is known that PPP1 plays a key role in mitosis where PPP1C isoforms are differentially targeted to specific subcellular structures. However, in previous studies, non-neuronal cells were used as a model system to study PPP1 distribution during the cell cycle [2,3]. In the studies here described, we used the neuronal-like cell line SH-SY5Y since PPP1 is a crucial protein in several neuronal functions.

In order to analyze the redistribution of PPP1CA and PPP1CC isoforms during mitosis we used nocodazole-blocked cells that were fixed in paraformaldeyde. The immunolocalization of PPP1C isoforms was achieved with specific PPP1C isoforms antibodies using confocal microscopy. Our results showed that both PPP1CA and PPP1CC are located in the cytoplasm and nucleus during interphase (Figure 1A). However, while PPP1CC accumulates in the nucleolus, PPP1CA is absent. During mitosis, both PPP1CA (Figure 1B) and PPP1CC (data not shown) are distributed throughout the cell excluding the chromosomal area and are also located in the mitotic spindle and in the mid body. Another interesting aspect is the co-localization of PPP1CA and gamma-tubulin to specific areas of the centrosomes (Figure 1C). Our results indicate that, in neuronal-like cells, PPP1C is redistributed during the cell cycle, suggesting that it is a crucial protein for mitosis. These results are in agreement with previous reports in non-neuronal cell lines.

\section{References}

1. Ceulemans and Bollen, Physiological Reviews 84 (1): 1-39, 2004

2. Andreassen et al., Journal of Cell Biology 141: 1207-1215, 1998

3. Trinkle-Mulcahy et al., Molecular Biology of the Cell 14:107-117, 2003

Acknowledgements: This work was supported by the Fundação para a Ciência e Tecnologia of the Portuguese Ministry of Science and Technology, the European Union (cNeupro), Center for Cell Biology - University of Aveiro, REEQ/1023/BIO/2005, PTDC/QUI-BIQ/101317/2008, POCTI/BIA$\mathrm{BCM} / 58469 / 2004$, and the scholarship awarded to MS, SFRH/BD/65353/2009. 

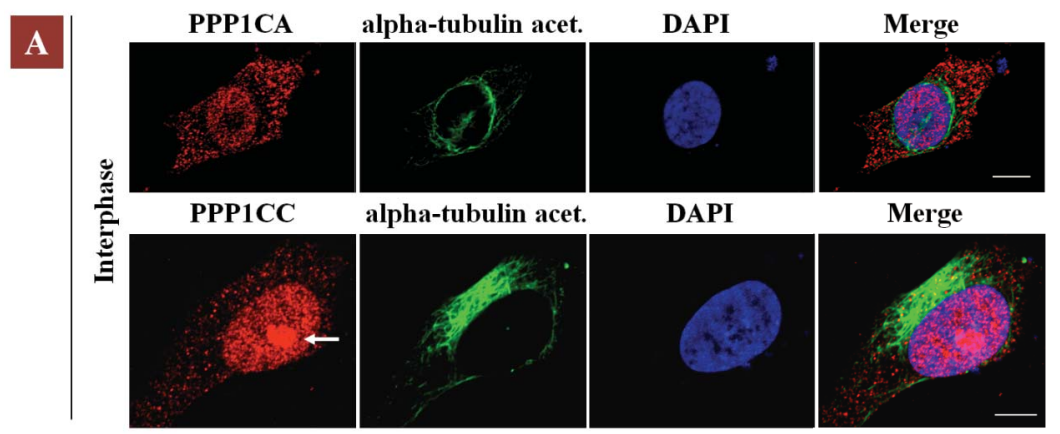

B

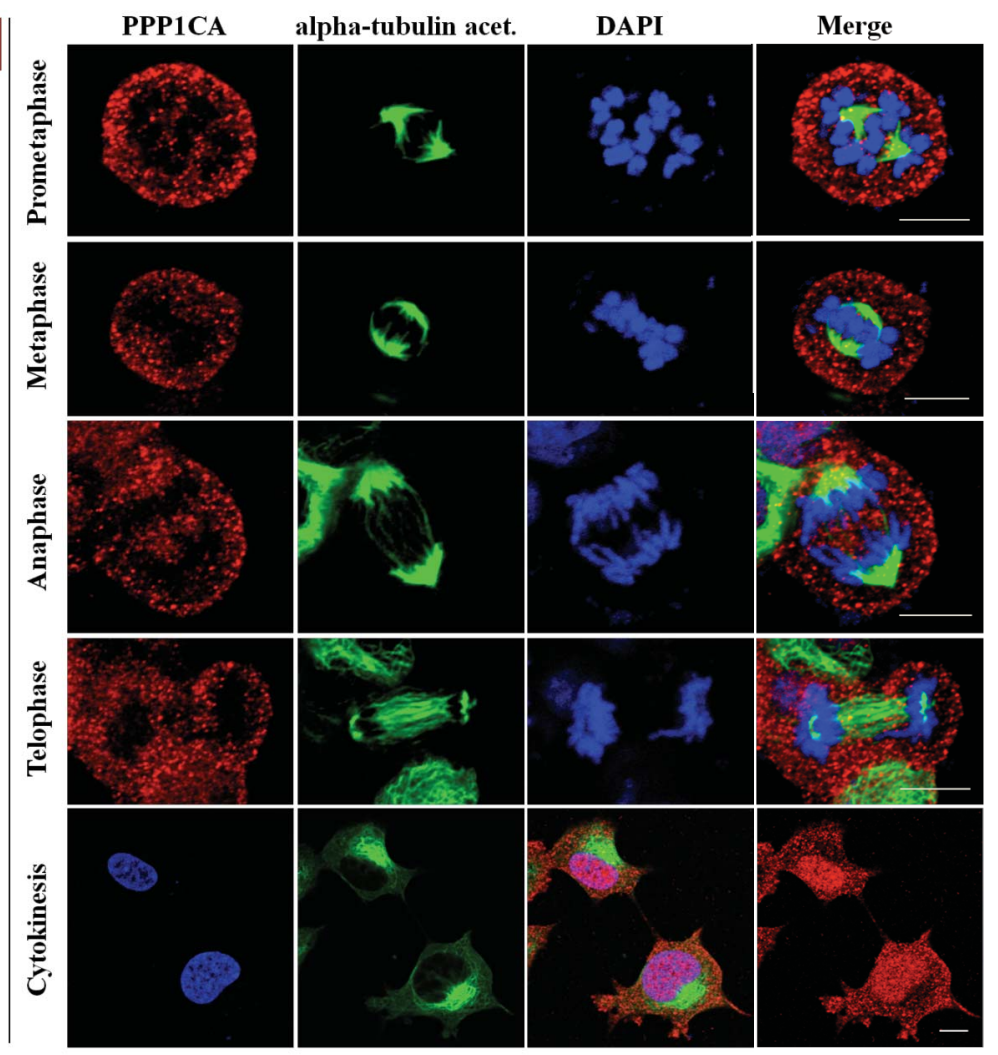

C

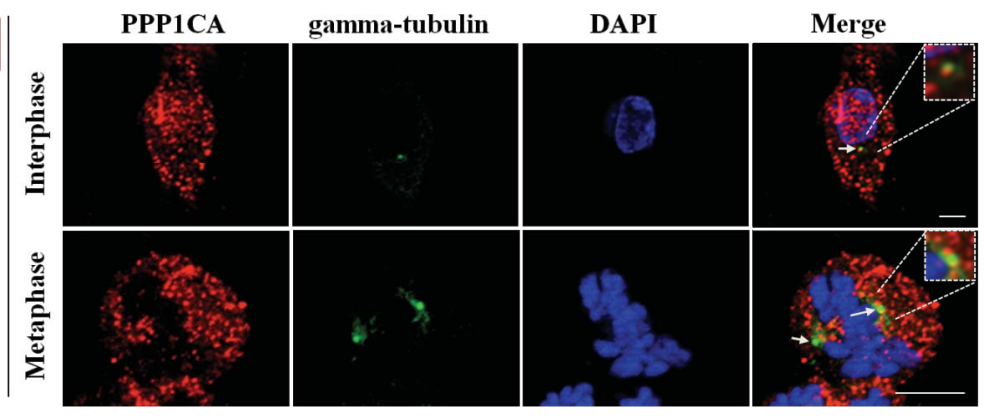

Figure 1. Subcellular distribution of PPP1CA and PPP1CC during the cell cycle in SH-S5Y5 cells. A - Immunolocalization of PPP1CA and PPP1CC during interphase. The arrow indicates the nucleolus. B - Immunolocalization of PPP1CA during different mitosis phases. C - Co-localization of PPP1CA and gamma-tubulin. The higher magnification view shows in more detail the co-localization of PPP1CA and gamma-tubulin in centrosomes. The arrows indicate the centrosomes. Cells were blocked in mitosis using $100 \mathrm{ng} / \mathrm{ml}$ Nocodazole. Specific primary antibodies for endogenous PPP1C isoforms were detected with Alexa Fluor 594 conjugated secondary antibody (red), alpha-tubulin acetylated (alpha-tubulin acet.) and gamma-tubulin were detected with Fluorescein conjugated secondary antibody (green). DNA was stained with DAPI (blue). Photographs were acquired using a LSM 510-Meta confocal microscope. Scale bars, $10 \mu \mathrm{m}$. 